\title{
Intuitionistic fuzzy transport equation
}

\author{
Zineb Belhallaj $^{1}$, Said Melliani ${ }^{2}$, M'hamed Elomari ${ }^{3}$ \\ and Lalla Saadia Chadli ${ }^{4}$ \\ ${ }^{1}$ LMACS, Sultan Moulay Slimane University \\ BP 523, 23000, Beni Mellal, Morocco \\ e-mail: zineb.belhallaj@gmail.com \\ 2 LMACS, Sultan Moulay Slimane University \\ BP 523, 23000, Beni Mellal, Morocco \\ e-mail: s.mellianidusms.ma \\ ${ }^{3}$ LMACS, Sultan Moulay Slimane University \\ BP 523, 23000, Beni Mellal, Morocco \\ e-mail: m.elomari@usms.ma \\ ${ }^{4}$ LMACS, Sultan Moulay Slimane University \\ BP 523, 23000, Beni Mellal, Morocco \\ e-mail: sa.chadli@yahoo.fr
}

Received: 16 May 2021

\begin{abstract}
In the present paper, we use the generalized differentiability concept to study the intuitionistic fuzzy transport equation. We consider transport equation in the homogeneous and non-homogeneous cases with intuitionistic fuzzy initial condition. To illustrate the results, we will solve an advection equation using the finite difference method.
\end{abstract}

Keywords: Intuitionistic fuzzy differential equations, Intuitionistic fuzzy transport equation, Finite difference method.

2020 Mathematics Subject Classification: 03E72, 35Q49.

\section{Introduction}

The Intuitionistic fuzzy differential equations (IFDE) have drawn much attention from scientists and engineers because of its applicability in various fields, as artificial intelligence, robotics, fluid mechanics, heat and mass transfer, economics and social sciences for modeling and solving their 
respective problems. The concept of Intuitionistic Fuzzy Sets (IFS) are a significant part of the fuzzy set theory [11]. In 1983 Atanassov generalized the fuzzy set theory by presenting the basic elements of intuitionistic fuzzy sets [1-3]. The theory of ordinary and partial fuzzy differential equations has been fabulously studied by several authors such as Seikkala [10], Bertone [5], B. Bede [4] and J. Prakash [9]. The existence and uniqueness of the solution of a differential equation with intuitionistic fuzzy data has been discussed in [6]. In this paper, we intend to study the effect of uncertainties on mass distribution. We emphasize on the intuitionistic fuzzy transport equation in homogeneous and non-homogeneous cases.

This manuscript is organized as follows. In Section 2, a few basic results on intuitionistic fuzzy sets and the metric space, which have been examined in $[7,8]$. In Section 3, we study homogeneous transport equations. In Section 4, the solution of non-homogeneous equation is given. The transport equation by non-precise speed is discussed in Section 5. Solving numerically the intuitionistic fuzzy advection equation by difference finite method is illustrated in Section 6 . Finally, a conclusion is drawn in Section 7.

\section{Preliminaries}

In this section, we present some definitions and introduce the necessary notation, which will be used throughout the paper.

\subsection{Intuitionistic fuzzy sets}

An intuitionistic fuzzy set $A \in X$ is given by

$$
A=\left\{\left(x, u_{A}(x), v_{A}(x)\right) \mid x \in X\right\},
$$

where the function $u_{A}(x), v_{A}(x): X \rightarrow[0,1]$ define respectively the degree of membership and degree of non-membership of the element $x \in X$ to the set $A$, which is a subset of $X$, and for every $x \in X, 0 \leq u(x)+v(x) \leq 1$. Obviously, every fuzzy set has the form

$$
\left\{\left(x, u_{A}(x), u_{A^{c}}(x)\right) \mid x \in X\right\} .
$$

For each intuitionistic fuzzy set $A \in X$, we call

$$
\pi_{A}(x)=1-u(x)-v(x)
$$

the degree of non-determinacy (uncertainty) of the element $x \in X$ to the intuitionistic fuzzy set $A$.

\subsection{Intuitionistic fuzzy numbers}

An element $\langle u, v\rangle$ of $\mathbb{F}_{1}$ is said to be an intuitionistic fuzzy number if it satisfies the following conditions:

(i) $\langle u, v\rangle$ is normal, i.e., there exists $x_{0}, x_{1} \in \mathbb{R}$ such that $u\left(x_{0}\right)=0$ and $v\left(x_{1}\right)=1$. 
(ii) The membership function $u$ is fuzzy convex, i.e.,

$$
u\left(\lambda x_{1}+(1-\lambda) x_{2}\right) \geq \min \left(u\left(x_{1}\right), u\left(x_{2}\right)\right)
$$

(iii) The non-membership function $v$ is fuzzy concave, i.e.,

$$
v\left(\lambda x_{1}+(1-\lambda) x_{2}\right) \leq \max \left(v\left(x_{1}\right), v\left(x_{2}\right)\right) .
$$

(iv) $u$ is upper semi-continuous and $v$ is lower semi-continuous.

(v) $\operatorname{Supp}\langle u, v\rangle=\operatorname{cl}\{x \in \mathbb{R}: \mid v(x)<1\}$ is bounded.

So we denote the collection of all intuitionistic fuzzy numbers by $\mathbb{F}_{1}$.

For $\alpha \in[0,1]$ and $\langle u, v\rangle \in \mathbb{F}^{1}$, the upper and lower $\alpha$-cuts of $\langle u, v\rangle$ are defined by

$$
\begin{aligned}
& {[\langle u, v\rangle]^{\alpha}=\{x \in \mathbb{R}: v(x) \leq 1-\alpha\}} \\
& {[\langle u, v\rangle]_{\alpha}=\{x \in \mathbb{R}: u(x) \geq \alpha\}}
\end{aligned}
$$

Remark 2.1. If $\langle u, v\rangle \in \mathbb{F}_{1}$, so we can see $[\langle u, v\rangle]_{\alpha}$ as $[u]^{\alpha}$ and $[\langle u, v\rangle]^{\alpha}$ as $[1-v]^{\alpha}$ in the fuzzy case.

Definition 2.2. Let $\kappa=\left(u_{\kappa}, v_{\kappa}\right), \kappa_{1}=\left(u_{\kappa_{1}}, v_{\kappa_{1}}\right)$ and $\kappa_{2}=\left(u_{\kappa_{2}}, v_{\kappa_{2}}\right)$ be three intuitionistic fuzzy numbers, then

1. $\kappa_{1} \bigoplus \kappa_{2}=\left(1-\left(1-u_{\kappa_{1}}\right)\left(1-u_{\kappa_{2}}\right), v_{\kappa_{1}} v_{\kappa_{2}}\right)$.

2. $\kappa_{1} \otimes \kappa_{2}=\left(u_{\kappa_{1}} u_{\kappa_{2}}, 1-\left(1-v_{\kappa_{1}}\right)\left(1-v_{\kappa_{2}}\right)\right)$.

3. $\lambda \kappa=\left(1-\left(1-u_{\kappa}\right)^{\lambda}, v_{\kappa}^{\lambda}\right), \quad \lambda>0$.

4. $\kappa^{\lambda}=\left(u_{\kappa}^{\lambda}, 1-\left(1-v_{\kappa}\right)^{\lambda}\right), \quad \lambda>0$.

We define $0_{(1,0)} \in \mathbb{F}_{1}$ as

$$
0_{(1,0)}(t)= \begin{cases}(1,0), & \text { if } t=0 . \\ (0,1), & \text { if } t \neq 0 .\end{cases}
$$

Let $\langle u, v\rangle,\left\langle u^{\prime}, v^{\prime}\right\rangle \in \mathbb{F}_{1}$ and $\lambda \in \mathbb{R}$. We define the following operations:

$$
\begin{gathered}
\left(\langle u, v\rangle \oplus\left\langle u^{\prime}, v^{\prime}\right\rangle\right)(z)=\left(\sup _{z=x+y} \min \left(u(x), u^{\prime}(y)\right), \inf _{z=x+y} \max \left(v(x), v^{\prime}(y)\right)\right), \\
\lambda\langle u, v\rangle= \begin{cases}\langle\lambda u, \lambda v\rangle, & \text { if } \lambda \neq 0 . \\
0_{(1,0)}, & \text { if } \lambda=0 .\end{cases}
\end{gathered}
$$

For $\langle u, v\rangle,\langle z, w\rangle \in \mathbb{F}_{1}$ and $\lambda \in \mathbb{R}$, the addition and scalar-multiplication are defined as follows

$$
\begin{array}{ll}
{[\langle u, v\rangle \oplus\langle z, w\rangle]^{\alpha}=[\langle u, v\rangle]^{\alpha}+[\langle z, w\rangle]^{\alpha},} & {[\lambda\langle z, w\rangle]^{\alpha}=\lambda[\langle z, w\rangle]^{\alpha},} \\
{[\langle u, v\rangle \oplus\langle z, w\rangle]_{\alpha}=[\langle u, v\rangle]_{\alpha}+[\langle z, w\rangle]_{\alpha},} & {[\lambda\langle z, w\rangle]_{\alpha}=\lambda[\langle z, w\rangle]_{\alpha} .}
\end{array}
$$


Definition 2.3. Let $\langle u, v\rangle$ be an element of $\mathbb{F}_{1}$ and $\alpha \in[0.1]$, we define the following sets:

$$
\begin{aligned}
& {[\langle u, v\rangle]_{l}^{+}(\alpha)=\inf \{x \in \mathbb{R} \mid u(x) \geq \alpha\}, \quad[\langle u, v\rangle]_{r}^{+}(\alpha)=\sup \{x \in \mathbb{R} \mid u(x) \geq \alpha\},} \\
& {[\langle u, v\rangle]_{l}^{-}(\alpha)=\inf \{x \in \mathbb{R} \mid v(x) \leq 1-\alpha\}, \quad[\langle u, v\rangle]_{r}^{-}(\alpha)=\sup \{x \in \mathbb{R} \mid v(x) \leq 1-\alpha\} .}
\end{aligned}
$$

Remark 2.4. $[\langle u, v\rangle]_{\alpha}=\left[[\langle u, v\rangle]_{l}^{+}(\alpha),[\langle u, v\rangle]_{r}^{+}(\alpha)\right],[\langle u, v\rangle]^{\alpha}=\left[[\langle u, v\rangle]_{l}^{-}(\alpha),[\langle u, v\rangle]_{r}^{-}(\alpha)\right]$.

Proposition 2.5. For all $\alpha, \beta \in[0,1]$ and $\langle u, v\rangle \in \mathbb{F}_{1}$

(i) $[\langle u, v\rangle]_{\alpha} \subset[\langle u, v\rangle]^{\alpha}$.

(ii) $[\langle u, v\rangle]_{\alpha}$ and $[\langle u, v\rangle]^{\alpha}$ are nonempty compact convex sets in $\mathbb{R}$.

(iii) If $\alpha \leq \beta$, then $[\langle u, v\rangle]_{\beta} \subset[\langle u, v\rangle]_{\alpha}$ and $[\langle u, v\rangle]^{\beta} \subset[\langle u, v\rangle]^{\alpha}$.

(iv) If $\alpha_{n} \nearrow \alpha$, then $[\langle u, v\rangle]_{\alpha}=\cap_{n}[\langle u, v\rangle]_{\alpha_{n}}$ and $[\langle u, v\rangle]^{\alpha}=\cap_{n}[\langle u, v\rangle]^{\alpha_{n}}$.

Let $M$ be an arbitrary set and $\alpha \in[0,1]$. We denote by

$$
M_{\alpha}=\{x \in \mathbb{R}: u(x) \geq \alpha\} \text { and } M^{\alpha}=\{x \in \mathbb{R}: v(x) \leq 1-\alpha\} .
$$

Lemma 2.6. Let $\left\{M_{\alpha}, \alpha \in[0,1]\right\}$ and $\left\{M^{\alpha}, \alpha \in[0,1]\right\}$ be two families of $\mathbb{R}$ which satisfy (i)-(iv) in Proposition 2.5. If $u$ and $v$ are defined by

$$
\begin{aligned}
& u(x)=\left\{\begin{array}{ll}
0, & \text { if } x \notin M_{0} \\
\sup \left\{\alpha \in[0,1]: x \in M_{\alpha}\right\}, & \text { if } x \in M_{0}
\end{array},\right. \\
& v(x)=\left\{\begin{array}{ll}
1, & \text { if } x \notin M_{0} \\
1-\sup \left\{\alpha \in[0,1]: x \in M^{\alpha}\right\}, & \text { if } x \in M_{0}
\end{array},\right.
\end{aligned}
$$

then $\langle u, v\rangle \in \mathbb{F}_{1}$.

Lemma 2.7. A mapping $d: \mathbb{F}_{1} \times \mathbb{F}_{1} \rightarrow \mathbb{R}$ is said to be an intuitionistic fuzzy metric on $\mathbb{F}_{1}$ if it satisfies the following conditions:

1. $d\left(\left\langle u_{1}, v_{1}\right\rangle,\left\langle u_{2}, v_{2}\right\rangle\right) \geq 0, \forall\left\langle u_{1}, v_{1}\right\rangle,\left\langle u_{2}, v_{2}\right\rangle \in \mathbb{F}_{1}$.

2. $d\left(\left\langle u_{1}, v_{1}\right\rangle,\left\langle u_{2}, v_{2}\right\rangle\right)=0$ iff $\left\langle u_{1}, v_{1}\right\rangle=\left\langle u_{2}, v_{2}\right\rangle$.

3. $d\left(\left\langle u_{1}, v_{1}\right\rangle,\left\langle u_{2}, v_{2}\right\rangle\right)=d\left(\left\langle u_{2}, v_{2}\right\rangle,\left\langle u_{1}, v_{1}\right\rangle\right) \forall\left\langle u_{1}, v_{1}\right\rangle,\left\langle u_{2}, v_{2}\right\rangle \in \mathbb{F}_{1}$.

4. $d\left(\left\langle u_{1}, v_{1}\right\rangle,\left\langle u_{3}, v_{3}\right\rangle\right) \leq d\left(\left\langle u_{1}, v_{1}\right\rangle,\left\langle u_{2}, v_{2}\right\rangle\right)+d\left(\left\langle u_{2}, v_{2}\right\rangle,\left\langle u_{3}, v_{3}\right\rangle\right), \forall\left\langle u_{1}, v_{1}\right\rangle,\left\langle u_{2}, v_{2}\right\rangle,\left\langle u_{3}, v_{3}\right\rangle \in$ $\mathbb{F}_{1}$.

On the space $\mathbb{F}_{1}$ we will consider the following metric,

$$
\begin{aligned}
d_{\infty}(\langle u, v\rangle,\langle z, w\rangle) & =\frac{1}{4} \sup _{0<\alpha \leq 1}\left\|\langle u, v\rangle_{r}^{+}(\alpha)-\langle z, w\rangle_{r}^{+}(\alpha)\right\| \\
& +\frac{1}{4} \sup _{0<\alpha \leq 1}\left\|\langle u, v\rangle_{l}^{+}(\alpha)-\langle z, w\rangle_{l}^{+}(\alpha)\right\| \\
& +\frac{1}{4} \sup _{0<\alpha \leq 1}\left\|\langle u, v\rangle_{r}^{-}(\alpha)-\langle z, w\rangle_{r}^{-}(\alpha)\right\| \\
& +\frac{1}{4} \sup _{0<\alpha \leq 1}\left\|\langle u, v\rangle_{l}^{-}(\alpha)-\langle z, w\rangle_{l}^{-}(\alpha)\right\|,
\end{aligned}
$$

where $\|$.$\| denotes the usual Euclidean norm in \mathbb{R}^{n}$. 
Proposition 2.8 ([7]). $\left(\mathbb{F}_{1}, d_{p}\right)$ is a metric space.

Definition 2.9. The generalized Hukuhara difference of two fuzzy numbers $\langle u, v\rangle,\left\langle u^{\prime}, v^{\prime}\right\rangle \in \mathbb{F}_{1}$ is defined as follows

$$
\langle u, v\rangle \ominus_{g H}\left\langle u^{\prime}, v^{\prime}\right\rangle=\langle w, z\rangle \Leftrightarrow\left\{\begin{array}{l}
\langle u, v\rangle=\left\langle u^{\prime}, v^{\prime}\right\rangle+\langle w, z\rangle, \text { or } \\
\left\langle u^{\prime}, v^{\prime}\right\rangle=\langle u, v\rangle+(-1)\langle w, z\rangle .
\end{array}\right.
$$

Definition 2.10. Let $F:(a, b) \rightarrow W^{1}$ and $x_{0} \in(a, b)$. It is said that $F$ is strongly generalized differentiable on $x_{0}$, if $\exists F^{\prime}+\left(x_{0}\right), \exists F^{\prime}-\left(x_{0}\right) \in E^{1}$, such that

(i) for all $h>0$ sufficiently small, $\exists F^{+}\left(x_{0}+h\right)-F^{+}\left(x_{0}\right), F^{+}\left(x_{0}\right)-F^{+}\left(x_{0}-h\right)$ and the limits (in the metric D)

$$
\lim _{h \rightarrow 0} \frac{F^{+}\left(x_{0}+h\right)-F^{+}\left(x_{0}\right)}{h}=\lim _{h \rightarrow 0} \frac{F^{+}\left(x_{0}\right)-F^{+}\left(x_{0}-h\right)}{h}=F^{\prime}\left(x_{0}\right)
$$

or

(ii) for all $h>0$ sufficiently small, $\exists F^{+}\left(x_{0}\right)-F^{+}\left(x_{0}+h\right), F^{+}\left(x_{0}-h\right)-F^{+}\left(x_{0}\right)$ and the limits

$$
\lim _{h \rightarrow 0} \frac{F^{+}\left(x_{0}\right)-F^{+}\left(x_{0}+h\right)}{-h}=\lim _{h \rightarrow 0} \frac{F^{+}\left(x_{0}-h\right)-F^{+}\left(x_{0}\right)}{-h}=F^{\prime}\left(x_{0}\right)
$$

or

(iii) for all $h>0$ sufficiently small, $\exists F^{+}\left(x_{0}+h\right)-F^{+}\left(x_{0}\right), F^{+}\left(x_{0}-h\right)-F^{+}\left(x_{0}\right)$ and the limitis

$$
\lim _{h \rightarrow 0} \frac{F^{+}\left(x_{0}\right)-F^{+}\left(x_{0}-h\right)}{h}=\lim _{h \rightarrow 0} \frac{F^{+}\left(x_{0}-h\right)-F^{+}\left(x_{0}\right)}{-h}=F^{+}\left(x_{0}\right)
$$

or

(iv) for all $h>0$ sufficiently small, $\exists F^{+}\left(x_{0}\right)-F^{+}\left(x_{0}+h\right), F^{+}\left(x_{0}\right)-F^{+}\left(x_{0}-h\right)$ and the limits

$$
\lim _{h \rightarrow 0} \frac{F^{+}\left(x_{0}\right)-F^{+}\left(x_{0}+h\right)}{-h}=\frac{F^{+}\left(x_{0}\right)-F^{+}\left(x_{0}-h\right)}{-h}=F^{\prime}\left(x_{0}\right) .
$$

Lemma 2.11. Let $f: \mathbb{R} \times(0, \infty) \rightarrow \mathbb{F}^{1}$ be strongly generalized differentiable with respect to $x$. Suppose there exists a continuous real-valued function $g(x, t)$ such that $D\left(\frac{\partial f}{\partial} x(x, t), \chi_{0}\right)$ $\leq g(x, t)$ for $x \in \mathbb{R}, t \geq 0$. Then $F(x, t)=\int_{0}^{t} f(x, s) d s$ is strongly generalized differentiable with respect to $x$ and we have $\frac{\partial F}{\partial x}(x, t)=\int_{0}^{t} \frac{\partial f}{\partial x}(x, s) d s$.

Proof. Let $f: \mathbb{R} \times(0, \infty) \rightarrow \mathbb{F}_{1}$ with $[f(x, t)]^{\alpha}=\left[f_{l}^{-}(\alpha)(x, t), f_{r}^{-}(\alpha)(x, t)\right]$ and $[f(x, t)]_{\alpha}=$ $\left[f_{l}^{+}(\alpha)(x, t), f_{r}^{+}(\alpha)(x, t)\right]$ be (i)-differentiable. We first notice that due to the our assumption we have the following derivatives uniformly with respect to $\alpha$.

$$
\begin{aligned}
\frac{\partial}{\partial x} \int_{0}^{t} f_{l}^{-}(\alpha)(x, s) d s & =\int_{0}^{t} \frac{\partial f_{l}^{-}(\alpha)}{\partial x}(x, s) d s, & \frac{\partial}{\partial x} \int_{0}^{t} f_{r}^{-}(\alpha)(x, s) d s & =\int_{0}^{t} \frac{\partial f_{r}^{-}(\alpha)}{\partial x}(x, s) d s, \\
\frac{\partial}{\partial x} \int_{0}^{t} f_{l}^{+}(\alpha)(x, s) d s & =\int_{0}^{t} \frac{\partial f_{l}^{+}(\alpha)}{\partial x}(x, s) d s, & \frac{\partial}{\partial x} \int_{0}^{t} f_{r}^{+}(\alpha)(x, s) d s & =\int_{0}^{t} \frac{\partial f_{r}^{+}(\alpha)}{\partial x}(x, s) d s .
\end{aligned}
$$


This fact yields:

$$
\begin{aligned}
\lim _{h \rightarrow 0} \frac{F(x+h, t) \ominus F(x, t)}{h} & =\lim _{h \rightarrow 0} \frac{\int_{0}^{t} f(x+h, s) d s \ominus \int_{0}^{t} f(x, s) d s}{h} \\
& =\lim _{h \rightarrow 0} \frac{\int_{0}^{t}(f(x+h, s) \ominus f(x, s)) d s}{h} \\
& =\int_{0}^{t} \lim _{h \rightarrow 0} \frac{f(x+h, s) \ominus f(x, s)}{h} d s \\
& =\int_{0}^{t} \frac{\partial f}{\partial x}(x, s) d s .
\end{aligned}
$$

It is easy to show for $F(x, t) \ominus F(x-h, t)$.

In a similar way, we can prove the assertion for (ii)-differentiability.

Lemma 2.12. Let $f: \mathbb{R} \times(0, \infty) \rightarrow \mathbb{F}_{1}$ be a continuous intuitionistic fuzzy-valued function and $b \in(0, \infty)$. Then

$$
\lim _{h \rightarrow 0} \frac{1}{h} \int_{t}^{t+h} f(x+(t-s) b+b h, s) d s=f(x, t) .
$$

Proof. Let $(x, t) \in \mathbb{R} \times(0, \infty)$, for given $\varepsilon>0$ there exists $\delta_{1}>0$ such that for all $0 \leq \alpha \leq 1$ and $|x-y|<\delta_{1},|t-s|<\delta_{1}$, we have $\left|f_{l}^{-}(\alpha)(y, t)-f_{l}^{-}(\alpha)(x, s)\right|<\varepsilon,\left|f_{r}^{-}(\alpha)(y, t)-f_{r}^{-}(\alpha)(x, s)\right|<\varepsilon$, $\left|f_{l}^{+}(\alpha)(y, t)-f_{l}^{+}(\alpha)(x, s)\right|<\varepsilon$ and $\left|f_{r}^{+}(\alpha)(y, t)-f_{r}^{+}(\alpha)(x, s)\right|<\varepsilon$.

Let $\delta=\min \left\{\frac{\delta_{1}}{2 b}, \delta_{1}\right\}$. For $|h| \leq \delta$, we have

$$
\frac{1}{h} \int_{t}^{t+h}\left|f_{l}^{-}(\alpha)(x+(t-s) b+h b, s)-f_{l}^{-}(\alpha)(x, t)\right| d s \leq \varepsilon,
$$

and the same result is valid for $f_{r}^{-}(\alpha), f_{l}^{+}(\alpha)$ and $f_{r}^{+}(\alpha)$ for all $\alpha \in[0,1]$. It shows that

$$
\lim _{h \rightarrow 0} D\left(\frac{1}{h} \int_{t}^{t+h} f(x+(t-s) b+b h, s) d s, f(x, t)\right)=0 .
$$

This completes the proof.

It is well-known that an intuitionistic function $f:(a, b) \rightarrow \mathbb{F}_{1}$ is (i)-differentiable if and only if the functions $[f]^{\alpha},[f]_{\alpha}$ are continuously differentiable with respect to $x$, uniformly with respect to $\alpha \in[0,1]$, provided that $\left[\left(f_{l}^{-}(\alpha)\right)^{\prime}(x),\left(f_{r}^{-}(\alpha)\right)^{\prime}(x)\right]$ and $\left[\left(f_{l}^{+}(\alpha)\right)^{\prime}(x),\left(f_{r}^{+}(\alpha)\right)^{\prime}(x)\right]$ define an intuitionistic fuzzy number $f^{\prime}(x) \in \mathbb{F}_{1}$.

Similarly, $f$ is (ii)-differentiable if and only if the functions $[f]^{\alpha},[f]_{\alpha}$ are continuously differentiable with respect to $x$, uniformly with respect to $\alpha \in[0,1]$, provided that $\left[\left(f_{r}^{-}(\alpha)\right)^{\prime}(x)\right.$, $\left.\left(f_{l}^{-}(\alpha)\right)^{\prime}(x)\right]$ and $\left[\left(f_{r}^{+}(\alpha)\right)^{\prime}(x),\left(f_{l}^{+}(\alpha)\right)^{\prime}(x)\right]$ define an intuitionistic fuzzy number $f^{\prime}(x) \in \mathbb{F}_{1}$.

In the following, we present a result only for existence of Hukuhara differences related to strongly generalized differentiability.

Theorem 2.13 ([2]). Let $f:(a, b) \rightarrow \mathbb{F}_{1}$ be such that $[f]^{\alpha}=\left[f_{l}^{-}(\alpha), f_{r}^{-}(\alpha)\right]$ and $[f]_{\alpha}=\left[f_{l}^{+}(\alpha), f_{r}^{+}(\alpha)\right]$. Suppose that real valued functions $f_{l}^{-}(\alpha), f_{r}^{-}(\alpha), f_{l}^{+}(\alpha)$ and $f_{r}^{+}(\alpha)$ are differentiable with respect to $x$. 
1. If the intervals $\left[\left(f_{l}^{-}(\alpha)\right)^{\prime}(x),\left(f_{r}^{-}(\alpha)\right)^{\prime}(x)\right]$ and $\left[\left(f_{l}^{+}(\alpha)\right)^{\prime}(x),\left(f_{r}^{+}(\alpha)\right)^{\prime}(x)\right]$ for all $\alpha \in[0,1]$ and $x \in(a, b)$, determine valid $\alpha$-cuts of a fuzzy number, then the H-differences $f(x+h) \ominus f(x)$ and $f(x) \ominus f(x-h)$ exist for all $h>0$ sufficiently small.

2. If the intervals $\left[\left(f_{r}^{-}(\alpha)\right)^{\prime}(x),\left(f_{l}^{-}(\alpha)\right)^{\prime}(x)\right]$ and $\left[\left(f_{r}^{+}(\alpha)\right)^{\prime}(x),\left(f_{l}^{+}(\alpha)\right)^{\prime}(x)\right]$ for all $\alpha \in[0,1]$ and $x \in(a, b)$, determine valid $\alpha$-cuts of a fuzzy number, then the H-differences $f(x) \ominus f(x+h)$ and $f(x-h) \ominus f(x)$ exist for all $h>0$ sufficiently small.

\section{Homogenous transport equation}

In this section, we intend to study the transport of a mass without any source with an intuitionistic fuzzy initial value and a positive speed (it can be negative, too):

$$
\begin{cases}u_{t}=b u_{x} & \text { on } \mathbb{R} \times(0, \infty) . \\ u(x, 0)=g(x) . & \end{cases}
$$

where $g$ is intuitionistic fuzzy valued function, and $b$ is a constant real number.

We say that $u(x, t)$ is a solution of $(3.1)$ on $\mathbb{R} \times(0, \infty)$ if $u$ is strongly differentiable with respect to $x, t$ and it verifies (3.1).

Our method is based on the constructive method in which we introduce the solution and examine that it verifies the problem.

Theorem 3.1. Suppose $g$ is a strongly generalized differentiable function on $\mathbb{R}$. Then $u(x, t)=$ $g(x+b t)$ is a solution of (3.1), where $b$ is a constant positive real number.

Proof. First, let $g$ be (i)-differentiable, then $g(x+t b+t h) \ominus g(x+t b)$ and $g(x+t b) \ominus g(x+t b-b h)$ for $h$ sufficiently small exist and we have:

$$
\begin{aligned}
u_{t} & =\lim _{h \rightarrow 0} \frac{u(x, t+h) \ominus u(x, t)}{h}=\lim _{h \rightarrow 0} \frac{g(x+t b+b h) \ominus g(x+t b)}{h} \\
& =\lim _{h \rightarrow 0} b \frac{g(x+t b+b h) \ominus g(x+t b)}{b h}=b g^{\prime}(x+t b) .
\end{aligned}
$$

Similarly:

$$
\begin{aligned}
u_{t} & =\lim _{h \rightarrow 0} \frac{u(x, t) \ominus u(x, t-h)}{h}=\lim _{h \rightarrow 0} \frac{g(x+t b) \ominus g(x+t b-b h)}{h} \\
& =\lim _{h \rightarrow 0} b \frac{g(x+t b) \ominus g(x+t b-b h)}{b h}=b g^{\prime}(x+t b) .
\end{aligned}
$$

On the other hand, we have:

$$
u_{x}=\lim _{h \rightarrow 0} \frac{u(x+h, t) \ominus u(x, t)}{h}=\lim _{h \rightarrow 0} \frac{g(x+t b+h) \ominus g(x+t b)}{h}=g^{\prime}(x+t b) .
$$

In the same way, we find $\lim _{h \rightarrow 0} \frac{u(x, t) \ominus u(x-h, t)}{h}$. This proves the (i)-differentiability of $u$ with respect to $x$ and $t$.

It is clear that $u$ satisfies the equation and the initial condition.

Similarly, when $g$ is (ii)-differentiable we can show that it is a solution. 


\section{Nonhomogeneous transport equation}

In this section, we focus on the following non homogeneous intuitionistic fuzzy transport equation for which the second member and initial value can be intuitionistic fuzzy

$$
\left\{\begin{array}{l}
u_{t}=b u_{x}+a u \quad \text { on } \mathbb{R} \times(0, \infty) . \\
u(x, 0)=g(x)
\end{array},\right.
$$

where $g$ and $f$ are intuitionistic fuzzy valued functions.

Theorem 4.1. Let $u: R \times(0, \infty) \rightarrow \mathbb{F}_{1}$ be (i)-differentiable with respect to $x$, t and let $g: \mathbb{R} \rightarrow \mathbb{F}_{1}$ and let $a$ and $b$ be positive real numbers.

1. If $g$ is (i)-differentiable, then $u_{1}(x, t)=g(x+t b)+\int_{0}^{t} a u(x+(t-s) b, s) d s$ is $a$ (i)-differentiable solution of (4.1).

2. If $g$ is (ii)-differentiable, then $u_{2}(x, t)=g(x+t b) \ominus(-1) \int_{0}^{t} a u(x+(t-s) b, s) d s$ is $a$ (ii)-differentiable solution of (4.1) provided the H-difference exists.

Proof.

1. First we suppose that $g$ is (i)-differentiable. We show that $u_{1}(x, t)$ is (i)-differentiable and this solves the problem.

$$
\begin{aligned}
\frac{\partial u_{1}}{\partial x} & =\lim _{h \rightarrow 0} \frac{u_{1}(x+h, t) \ominus u_{1}(x, t)}{h} \\
& =\lim _{h \rightarrow 0} \frac{\left.\left(g(x+t b+h)+\int_{0}^{t} a u(x+h+(t-s) b, s) d s\right) \ominus\left(g(x+t b)+\int_{0}^{t} a u(x+(t-s) b, s) d s\right)\right)}{h} \\
& =\lim _{h \rightarrow 0} \frac{(g(x+t b+h) \ominus g(x+t b))}{h}+\lim _{h \rightarrow 0} \frac{\int_{0}^{t} a(u(x+h+(t-s) b, s) \ominus u(x+(t-s) b, s)) d s}{h} \\
& =g^{\prime}(x+t b)+\lim _{h \rightarrow 0} \frac{a}{h} \int_{0}^{t}((u(x+h+(t-s) b, s) \ominus u(x+(t-s) b, s)) d s . \\
\frac{\partial u_{1}}{\partial x} & \left.=g^{\prime}(x+t b)+\int_{0}^{t} a \frac{\partial}{\partial x} u(x+(t-s) b, s)\right) d s .
\end{aligned}
$$

For the derivative with respect to $t$, from Lemma 2.11, we have:

$$
\begin{aligned}
\frac{\partial u_{1}}{\partial t} & =\lim _{h \rightarrow 0} \frac{u_{1}(x, t+h) \ominus u_{1}(x, t)}{h} \\
& =\lim _{h \rightarrow 0} \frac{\left.\left(g(x+(t+h) b)+\int_{0}^{t} a u(x+(t+h-s) b, s) d s\right) \ominus\left(g(x+t b)+\int_{0}^{t} a u(x+(t-s) b, s) d s\right)\right)}{h} \\
& =b g^{\prime}(x+t b)+\lim _{h \rightarrow 0} \int_{0}^{t} \frac{a(u(x+(t-s) b+h b, s) \Theta u(x+(t-s) b, s)) d s}{h} \\
& +\lim _{h \rightarrow 0} \int_{t}^{t+h} \frac{a u(x+(t-s) b+h b, s) d s}{h} . \\
\frac{\partial u_{1}}{\partial t} & =b g^{\prime}(x+t b)+a \cdot b \int_{0}^{t} \frac{\partial u}{\partial x}(x+(t-s) b, s) d s+a u(x, t) .
\end{aligned}
$$

Therefore, $u_{1}$ is a (i)-differentiable solution of (4.1). 
2. Now let $g$ be (ii)-differentiable. From Lemma 2.12, $u_{2}$ is (ii)-differentiable with respect to $x$ and we have:

$$
\frac{\partial u_{2}}{\partial x}=g^{\prime}(x+t b)+(-1) \int_{0}^{t}-a \frac{\partial u}{\partial x}(x+(t-s) b, s) d s .
$$

Now, we compute (ii)-derivative of $u_{2}$ with respect to $t$, first we have:

$$
\begin{aligned}
u_{2}(x, t) \ominus u_{2}(x, t+h)= & {\left[g(x+t b) \ominus \int_{0}^{t}-a u(x+(t-s) b, s) d s\right] } \\
& \ominus\left[g(x+t b+b h) \ominus \int_{0}^{t+h}-a u(x+(t-s) b+b h, s) d s\right] \\
= & {[g(x+t b) \ominus g(x+t b+b h)] } \\
& +\left[\int_{0}^{t+h}-a u(x+(t-s) b+b h, s) d s \ominus \int_{0}^{t}-a u(x+(t-s) b, s) d s\right] \\
= & {[g(x+t b) \ominus g(x+t b+b h)]+\int_{0}^{t}-a u(x+(t-s) b+b h, s) d s } \\
& \ominus \int_{0}^{t}-a u(x+(t-s) b, s) d s+\int_{t}^{t+h}-f(x+(t-s) b+b h, s) d s .
\end{aligned}
$$

After multiplying by $-\frac{1}{h}$ and taking the limit as $h \rightarrow 0^{+}$, we obtain:

$$
\begin{aligned}
& \frac{\partial u_{2}}{\partial t}(x, t)=\lim _{h \rightarrow 0} \frac{u_{2}(x, t) \ominus u_{2}(x, t+h)}{-h} . \\
& \frac{\partial u_{2}}{\partial t}(x, t)=b g^{\prime}(x+t b)+a . b \int_{0}^{t} \frac{\partial u}{\partial x}(x+(t-s) b, s) d s+a . u(x, t) .
\end{aligned}
$$

\section{Equation by non-precise speed}

In this section, we study the following homogeneous intuitionistic fuzzy transport equation for which the speed and initial value can be intuitionistic fuzzy.

$$
\left\{\begin{array}{l}
u_{t}=b u_{x} \quad \text { on } \mathbb{R} \times(0, \infty) \\
u(x, 0)=c g(x)
\end{array},\right.
$$

where $b, c \in \mathbb{F}_{1}$ and $g$ is a real-valued function.

In the following theorem, using Zadeh's extension principle, we intuitionistically fuzzify the real function $g: \mathbb{R} \rightarrow \mathbb{R}$ to intuitionistic fuzzy function $\tilde{g}: \mathbb{F}_{1} \rightarrow \mathbb{F}_{1}$. Since $g$ is a continuous function, we have $[\tilde{g}(X)]^{\alpha}=g\left([X]^{\alpha}\right)$ and $[\tilde{g}(X)]_{\alpha}=g\left([X]_{\alpha}\right)$.

For instance, let $X=x+t b$ and $g$ be an increasing function. Then, $\forall \alpha \in[0,1]$ we have:

$$
[\tilde{g}(x+t b)]^{\alpha}=g\left([x+t b]^{\alpha}\right), \quad[\tilde{g}(x+t b)]_{\alpha}=g\left([x+t b]_{\alpha}\right) .
$$

So

$$
\begin{aligned}
& {[\tilde{g}(x+t b)]^{\alpha}=g\left([x+t b]^{\alpha}\right)=\left[g\left(x+t b_{l}^{-}(\alpha)\right), g\left(x+t b_{r}^{-}(\alpha)\right)\right], \text { and }} \\
& {[\tilde{g}(x+t b)]_{\alpha}=g\left([x+t b]_{\alpha}\right)=\left[g\left(x+t b_{l}^{+}(\alpha)\right), g\left(x+t b_{r}^{+}(\alpha)\right)\right] .}
\end{aligned}
$$


Theorem 5.1. Suppose $g \in C^{2}(\mathbb{R})$ is an integrable nonnegative monotone function and $b, c \in \mathbb{F}_{1}$. Let $\tilde{g}: \mathbb{F}_{1} \rightarrow \mathbb{F}_{1}$ be the Zadeh's extension of $g$. Consider:

$$
u: \mathbb{R} \times(0, \infty) \rightarrow \mathbb{F}_{1}, \quad u(x, t)=c \tilde{g}(x+t b) .
$$

(i) If $b, c \in \mathbb{F}_{1}^{+}$and $g, g^{\prime}$ are nondecreasing functions, then $u$ is (i)-differentiable with respect to both $t, x$ and it is a solution of (5.1);

(ii) If $b \in \mathbb{F}_{1}^{-}, c \in \mathbb{F}_{1}^{+}$and $g$ is nonincreasing and $g^{\prime}$ is nondecreasing function, then $u$ is (i)-differentiable with respect to t and (ii)-differentiable with respect to $x$ and satisfies (5.1);

(iii) If $b \in \mathbb{F}_{n}^{+}, c \in \mathbb{F}_{n}^{-}$and $g, g^{\prime}$ are nondecreasing functions, then $u$ is (i)-differentiable with respect to both $t, x$ and satisfies (5.1);

(iv) If $b, c \in \mathbb{F}_{n}^{-}$and $g$ is nonincreasing and $g^{\prime}$ is a decreasing function, then $u$ is (i)-differentiable with respect to $t$ and (ii)-differentiable with respect to $x$ and satisfies (5.1).

Proof. Case (i): It is easy to check that the $\alpha$-cuts:

$$
\begin{aligned}
& {[u]^{\alpha}=\left[c_{l}^{-}(\alpha) g\left(x+t b_{l}^{-}(\alpha)\right), c_{r}^{-}(\alpha) g\left(x+t b_{r}^{-}(\alpha)\right)\right],} \\
& {[u]_{\alpha}=\left[c_{l}^{+}(\alpha) g\left(x+t b_{l}^{+}(\alpha)\right), c_{r}^{+}(\alpha) g\left(x+t b_{r}^{+}(\alpha)\right)\right]}
\end{aligned}
$$

satisfy the conditions in case (i) of Theorem 2.13.

Indeed,

$$
\left[c_{l}^{-}(\alpha) b_{l}^{-}(\alpha) g^{\prime}\left(x+t b_{l}^{-}(\alpha)\right), c_{r}^{-}(\alpha) b_{r}^{-}(\alpha) g^{\prime}\left(x+t b_{r}^{-}(\alpha)\right)\right]
$$

and

$$
\left[c_{l}^{+}(\alpha) b_{l}^{+}(\alpha) g^{\prime}\left(x+t b_{l}^{+}(\alpha)\right), c_{r}^{+}(\alpha) b_{r}^{+}(\alpha) g^{\prime}\left(x+t b_{r}^{+}(\alpha)\right)\right]
$$

are valid $\alpha$-cuts of intuitionistic fuzzy number. Then the H-differences $u(x, t+h) \ominus u(x, t)$ and $u(x, t) \ominus u(x, t-h)$ exist.

In a similar way, since

$$
\left[c_{l}^{-}(\alpha) g^{\prime}\left(x+t b_{l}^{-}(\alpha)\right), c_{r}^{-}(\alpha) g^{\prime}\left(x+t b_{r}^{-}(\alpha)\right)\right]
$$

and

$$
\left[c_{l}^{+}(\alpha) g^{\prime}\left(x+t b_{l}^{+}(\alpha)\right), c_{r}^{+}(\alpha) g^{\prime}\left(x+t b_{r}^{+}(\alpha)\right)\right]
$$

form an intuitionistic fuzzy number, the H-differences $u(x+h, t) \ominus u(x, t)$ and $u(x, t) \ominus u(x-h, t)$ exist.

Now, we show that the following limits are uniform with respect to $\alpha \in[0,1]$.

$$
\begin{aligned}
& \lim _{h \rightarrow 0} \frac{c_{l}^{-}(\alpha) g\left(x+(t+h) b_{l}^{-}(\alpha)\right)-c_{l}^{-}(\alpha) g\left(x+t b_{l}^{-}(\alpha)\right)}{h}=c_{l}^{-}(\alpha) b_{l}^{-}(\alpha) g^{\prime}\left(x+t b_{l}^{-}(\alpha)\right) . \\
& \lim _{h \rightarrow 0} \frac{c_{r}^{-}(\alpha) g\left(x+(t+h) b_{r}^{-}(\alpha)\right)-c_{r}^{-}(\alpha) g\left(x+t b_{r}^{-}(\alpha)\right)}{h}=c_{r}^{-}(\alpha) b_{r}^{-}(\alpha) g^{\prime}\left(x+t b_{r}^{-}(\alpha)\right) . \\
& \lim _{h \rightarrow 0} \frac{c_{l}^{+}(\alpha) g\left(x+(t+h) b_{l}^{+}(\alpha)\right)-c_{l}^{+}(\alpha) g\left(x+t b_{l}^{+}(\alpha)\right)}{h}=c_{l}^{+}(\alpha) b_{l}^{+}(\alpha) g^{\prime}\left(x+t b_{l}^{+}(\alpha)\right) . \\
& \lim _{h \rightarrow 0} \frac{c_{r}^{+}(\alpha) g\left(x+(t+h) b_{r}^{+}(\alpha)\right)-c_{r}^{+}(\alpha) g\left(x+t b_{r}^{+}(\alpha)\right)}{h}=c_{r}^{+}(\alpha) b_{r}^{+}(\alpha) g^{\prime}\left(x+t b_{r}^{+}(\alpha)\right) . \\
& \lim _{h \rightarrow 0} \frac{c_{l}^{-}(\alpha) g\left(x+t b_{l}^{-}(\alpha)\right)-c_{l}^{-}(\alpha) g\left(x+(t-h) b_{l}^{-}(\alpha)\right)}{h}=c_{l}^{-}(\alpha) b_{l}^{-}(\alpha) g^{\prime}\left(x+t b_{l}^{-}(\alpha)\right) .
\end{aligned}
$$




$$
\begin{aligned}
& \lim _{h \rightarrow 0} \frac{c_{r}^{-}(\alpha) g\left(x+t b_{r}^{-}(\alpha)\right)-c_{r}^{-}(\alpha) g\left(x+(t-h) b_{r}^{-}(\alpha)\right)}{h}=c_{r}^{-}(\alpha) b_{r}^{-}(\alpha) g^{\prime}\left(x+t b_{r}^{-}(\alpha)\right) . \\
& \lim _{h \rightarrow 0} \frac{c_{l}^{+}(\alpha) g\left(x+t b_{l}^{+}(\alpha)\right)-c_{l}^{+}(\alpha) g\left(x+(t-h) b_{l}^{+}(\alpha)\right)}{h}=c_{l}^{+}(\alpha) b_{l}^{+}(\alpha) g^{\prime}\left(x+t b_{l}^{+}(\alpha)\right) . \\
& \lim _{h \rightarrow 0} \frac{c_{r}^{+}(\alpha) g\left(x+t b_{r}^{+}(\alpha)\right)-c_{r}^{+}(\alpha) g\left(x+(t-h) b_{r}^{+}(\alpha)\right)}{h}=c_{r}^{+}(\alpha) b_{r}^{+}(\alpha) g^{\prime}\left(x+t b_{r}^{+}(\alpha)\right) .
\end{aligned}
$$

For (5.2) we have

$$
\begin{aligned}
& \lim _{h \rightarrow 0} \sup _{\alpha \in[0,1]}\left|\frac{c_{l}^{-}(\alpha) g\left(x+(t+h) b_{l}^{-}(\alpha)\right)-c_{l}^{-}(\alpha) g\left(x+t b_{l}^{-}(\alpha)\right)}{h}-c_{l}^{-}(\alpha) b_{l}^{-}(\alpha) g^{\prime}\left(x+t b_{l}^{-}(\alpha)\right)\right| \\
= & \lim _{h \rightarrow 0} \sup _{\alpha \in[0,1]}\left|c_{l}^{-}(\alpha) b_{l}^{-}(\alpha) \| \frac{g\left(x+(t+h) b_{l}^{-}(\alpha)\right)-g\left(x+t b_{l}^{-}(\alpha)\right)}{h b_{l}^{-}(\alpha)}-g^{\prime}\left(x+t b_{l}^{-}(\alpha)\right)\right| \\
= & \lim _{h \rightarrow 0} \sup _{\alpha \in[0,1]}\left|c_{l}^{-}(\alpha) b_{l}^{-}(\alpha) \| g^{\prime}\left(x+t b_{l}^{-}(\alpha)+\zeta(h, \alpha)\right)-g^{\prime}\left(x+t b_{l}^{-}(\alpha)\right)\right| \\
= & \lim _{h \rightarrow 0} \sup _{\alpha \in[0,1]}\left|c_{l}^{-}(\alpha) b_{l}^{-}(\alpha) \| g^{\prime \prime}\left(x+t b_{l}^{-}(\alpha)+\eta(h, \alpha)\right) \zeta(h, \alpha)\right| \\
\leq & \lim _{h \rightarrow 0} c_{0} b_{0}^{2} M(x, t) h=0,
\end{aligned}
$$

where $\zeta(h, \alpha)$ is a point on the line segment between 0 and $h b_{l}^{-}(\alpha)$ and $\eta(h, \alpha)$ is a point on the line segment between 0 and $\zeta(h, \alpha)$. And also, $\left|b_{l}^{-}(\alpha)\right| \leq \max \left\{b_{l}^{-}(0), b_{r}^{-}(0)\right\}=b_{0}$ and $\left|c_{l}^{-}(\alpha)\right| \leq \max \left\{c_{l}^{-}(0), c_{r}^{-}(0)\right\}=c_{0}$, for all $\alpha \in[0,1]$. Then

$$
\begin{gathered}
\left.x+(t+h) b_{0} \leq x+t b_{l}^{-}(\alpha)+\eta(h, \alpha)\right) \leq x+(t+h) b_{0}, \\
\left|g^{\prime \prime}\left(x+t b_{l}^{-}(\alpha)+\eta(h, \alpha)\right) \leq M(x, t)\right| .
\end{gathered}
$$

This concludes that

$$
\left[u_{t}(x, t)\right]^{\alpha}=\left[c_{l}^{-}(\alpha) b_{l}^{-}(\alpha) g^{\prime}\left(x+t b_{l}^{-}(\alpha)\right), c_{r}^{-}(\alpha) b_{r}^{-}(\alpha) g^{\prime}\left(x+t b_{r}^{-}(\alpha)\right)\right]
$$

and

$$
\left[u_{t}(x, t)\right]_{\alpha}=\left[c_{l}^{+}(\alpha) b_{l}^{+}(\alpha) g^{\prime}\left(x+t b_{l}^{+}(\alpha)\right), c_{r}^{+}(\alpha) b_{r}^{+}(\alpha) g^{\prime}\left(x+t b_{r}^{+}(\alpha)\right)\right]
$$

Case (ii): According to Zadeh's extension principle, $\alpha$ level sets $u$ is defined as:

$$
\begin{aligned}
& {[u]^{\alpha}=\left[c_{l}^{-}(\alpha) g\left(x+t b_{l}^{+}(\alpha)\right), c_{r}^{-}(\alpha) g\left(x+t b_{r}^{+}(\alpha)\right)\right],} \\
& {[u]_{\alpha}=\left[c_{l}^{+}(\alpha) g\left(x+t b_{l}^{-}(\alpha)\right), c_{r}^{+}(\alpha) g\left(x+t b_{r}^{-}(\alpha)\right)\right] .}
\end{aligned}
$$

Also, since $g^{\prime}$ is a non-positive function, then

$$
\left[c_{l}^{-}(\alpha) b_{l}^{+}(\alpha) g^{\prime}\left(x+t b_{l}^{+}(\alpha)\right), c_{r}^{-}(\alpha) b_{r}^{+}(\alpha) g^{\prime}\left(x+t b_{r}^{+}(\alpha)\right)\right]
$$

and

$$
\left[c_{l}^{+}(\alpha) b_{l}^{-}(\alpha) g^{\prime}\left(x+t b_{l}^{-}(\alpha)\right), c_{r}^{+}(\alpha) b_{r}^{-}(\alpha) g^{\prime}\left(x+t b_{r}^{-}(\alpha)\right)\right]
$$

form an intuitionistic fuzzy number, by Theorem 2.13, the H-differences $u(x, t+h) \ominus u(x, t)$ and $u(x, t) \ominus u(x, t-h)$ exist. 
In a similar way, since $\left[c_{l}^{+}(\alpha) g^{\prime}\left(x+t b_{l}^{-}(\alpha)\right), c_{r}^{+}(\alpha) g^{\prime}\left(x+t b_{r}^{-}(\alpha)\right)\right]$ and $\left[c_{l}^{-}(\alpha) g^{\prime}\left(x+t b_{l}^{+}(\alpha)\right)\right.$, $\left.c_{r}^{-}(\alpha) g^{\prime}\left(x+t b_{r}^{+}(\alpha)\right)\right]$ form an intuitionistic fuzzy number, by the theorem, the H-differences $u(x, t) \ominus u(x+h, t)$ and $u(x-h, t) \ominus u(x, t)$ exist.

Case (iii): According to Zadeh's extension principle, $\alpha$ level sets $u$ is defined as:

$$
\begin{aligned}
& {[u]^{\alpha}=\left[c_{l}^{+}(\alpha) g\left(x+t b_{l}^{-}(\alpha)\right), c_{r}^{+}(\alpha) g\left(x+t b_{r}^{-}(\alpha)\right)\right],} \\
& {[u]_{\alpha}=\left[c_{l}^{-}(\alpha) g\left(x+t b_{l}^{+}(\alpha)\right), c_{r}^{-}(\alpha) g\left(x+t b_{r}^{+}(\alpha)\right)\right] .}
\end{aligned}
$$

Also, since $g^{\prime}$ is a nonnegative function, then:

$$
\left[c_{l}^{+}(\alpha) b_{l}^{-}(\alpha) g^{\prime}\left(x+t b_{l}^{-}(\alpha)\right), c_{r}^{+}(\alpha) b_{r}^{-}(\alpha) g^{\prime}\left(x+t b_{r}^{-}(\alpha)\right)\right]
$$

and

$$
\left[c_{l}^{-}(\alpha) b_{l}^{+}(\alpha) g^{\prime}\left(x+t b_{l}^{+}(\alpha)\right), c_{r}^{-}(\alpha) b_{r}^{+}(\alpha) g^{\prime}\left(x+t b_{r}^{+}(\alpha)\right)\right]
$$

form an intuitionistic fuzzy number, by Theorem 2.13, the H-differences $u(x, t+h) \ominus u(x, t)$ and $u(x, t) \ominus u(x, t-h)$ exist.

In a similar way, since

$$
\left[c_{l}^{-}(\alpha) g^{\prime}\left(x+t b_{l}^{+}(\alpha)\right), c_{r}^{-}(\alpha) g^{\prime}\left(x+t b_{r}^{+}(\alpha)\right)\right]
$$

and

$$
\left[c_{l}^{+}(\alpha) g^{\prime}\left(x+t b_{l}^{-}(\alpha)\right), c_{r}^{+}(\alpha) g^{\prime}\left(x+t b_{r}^{-}(\alpha)\right)\right]
$$

form an intuitionistic fuzzy number, by Theorem 2.13, the H-differences $u(x+h, t) \ominus u(x, t)$ and $u(x, t) \ominus u(x-h, t)$ exist.

Case (iv): According to Zadeh's extension principle, $\alpha$ level sets $u$ is defined as:

$$
\begin{aligned}
& {[u]^{\alpha}=\left[c_{l}^{-}(\alpha) g\left(x+t b_{l}^{-}(\alpha)\right), c_{r}^{-}(\alpha) g\left(x+t b_{r}^{-}(\alpha)\right)\right],} \\
& {[u]_{\alpha}=\left[c_{l}^{+}(\alpha) g\left(x+t b_{l}^{+}(\alpha)\right), c_{r}^{+}(\alpha) g\left(x+t b_{r}^{+}(\alpha)\right)\right] .}
\end{aligned}
$$

On the other hand, since $g^{\prime}$ is a nonpositive function, then

$$
\left[c_{l}^{-}(\alpha) b_{l}^{-}(\alpha) g^{\prime}\left(x+t b_{l}^{-}(\alpha)\right), c_{r}^{-}(\alpha) b_{r}^{-}(\alpha) g^{\prime}\left(x+t b_{r}^{-}(\alpha)\right)\right]
$$

and

$$
\left[c_{l}^{+}(\alpha) b_{l}^{+}(\alpha) g^{\prime}\left(x+t b_{l}^{+}(\alpha)\right), c_{r}^{+}(\alpha) b_{r}^{+}(\alpha) g^{\prime}\left(x+t b_{r}^{+}(\alpha)\right)\right]
$$

form a fuzzy number. By Theorem 2.13, the H-differences $u(x, t+h) \ominus u(x, t)$ and $u(x, t) \ominus u(x, t-h)$ exist. In a similar way, since

$$
\left[c_{l}^{+}(\alpha) g^{\prime}\left(x+t b_{l}^{+}(\alpha)\right), c_{r}^{-}(\alpha) g^{\prime}\left(x+t b_{r}^{-}(\alpha)\right)\right]
$$

and

$$
\left[c_{l}^{-}(\alpha) g^{\prime}\left(x+t b_{l}^{-}(\alpha)\right), c_{r}^{+}(\alpha) g^{\prime}\left(x+t b_{r}^{+}(\alpha)\right)\right]
$$

form a fuzzy number, the H-differences $u(x, t) \ominus u(x+h, t)$ and $u(x-h, t) \ominus u(x, t)$ exist. 


\section{Application}

Consider the advection equation (5.1) to study an example of the transport of a Gaussian curve along a channel, where $g(x)=\exp \left[-200\left(x-x_{c}\right)^{2}\right]$ and $x_{c}$ is the center of the curve at $t=0$, $b$ and $c \in \mathbb{F}_{1}^{+}$.

Then $u(x, t)=c \tilde{g}(x+t b)$ is the solution with $\alpha$-cuts.

To solve this equation we are going to use the finite difference method, so transmissive boundary conditions are employed at either of the domain, i.e.,

$$
\begin{aligned}
u_{-1} & =u_{1} . \\
u_{N+1} & =u_{N-1} .
\end{aligned}
$$

Assume that $\tilde{U}$ is an intuitionistic fuzzy function of the independent crisp variable $x$ and $t$. Subdivide the $x-t$ plane into sets of equal rectangles of sides $\delta x=h, \delta t=k$ by equally space grid lines parallel to $O y$, defined by $x_{i}=i h, i=0,1,2,3, \ldots, n$. And equally spaced grid lines parallel to $O x$, defined by $y_{j}=j k, j=0,1,2, \ldots, n$. Denote the value of $\tilde{U}$ at the representative mesh point $p(i h, j k)$ by $\tilde{U}_{p}=\tilde{U}(i h, j k)=\tilde{U}_{i j}$.

Also, denote the parametric form of the intuitionistic fuzzy number, $\tilde{U}_{i j}$ as follows $\tilde{U}_{i j}=\left[\left(\underline{U}_{i, j}, \bar{U}_{i, j}\right),\left(\underline{\underline{U}} i, j, \overline{\bar{U}}_{i, j}\right)\right]$, such that $\underline{U}_{i, j}=\left[U_{i, j}\right]_{l}^{+}(\alpha), \bar{U}_{i, j}=\left[U_{i, j}\right]_{r}^{+}(\alpha), \underline{\underline{U}} i, j=\left[U_{i, j}\right]_{l}^{-}(\alpha)$ and $\overline{\bar{U}}_{i, j}=\left[U_{i, j}\right]_{r}^{-}(\alpha)$.

We have $\left(D_{t}\right) U_{i, j}=\left[\left[\left(D_{t}\right) U_{i, j}\right]_{\alpha},\left[\left(D_{t}\right) U_{i, j}\right]^{\alpha}\right]$ and $\left(D_{x}\right) U_{i, j}=\left[\left[\left(D_{x}\right) U_{i, j}\right]_{\alpha},\left[\left(D_{x}\right) U_{i, j}\right]^{\alpha}\right]$, where

$$
\begin{aligned}
{\left[\left(D_{t}\right) U_{i, j}\right]_{\alpha}=\left[\left(D_{t}\right) U_{i, j}, \overline{\left(D_{t}\right) U_{i, j}}\right] ; } & {\left[\left(D_{t}\right) U_{i, j}\right]^{\alpha}=\left[\underline{\underline{\left(D_{t}\right) U_{i, j}}}, \overline{\overline{\left(D_{t}\right) U_{i, j}}}\right] } \\
{\left[\left(D_{x}\right) U_{i, j}\right]_{\alpha}=\left[\underline{\left(D_{x}\right) U_{i, j}}, \overline{\left(D_{x}\right) U_{i, j}}\right] ; } & {\left[\left(D_{x}\right) U_{i, j}\right]^{\alpha}=\left[\underline{\underline{\left(D_{x}\right) U_{i, j}}}, \overline{\overline{\left(D_{x}\right) U_{i, j}}}\right] . }
\end{aligned}
$$

Then, for $\left(D_{t}\right) \tilde{U}_{i j}$ at $p$ we have:

$$
\begin{array}{ll}
\underline{\left(D_{t}\right) U_{i, j}}=\frac{\underline{U}_{i, j+1}-\underline{U}_{i, j}}{k} ; & \overline{\left(D_{t}\right) U_{i, j}}=\frac{\bar{U}_{i, j+1}-\bar{U}_{i, j}}{k} \\
\underline{\underline{\left(D_{t}\right) U_{i, j}}}=\frac{\underline{\underline{U}}_{i, j+1}-\underline{\underline{U}}_{i, j}}{k} ; & \overline{\overline{\left(D_{t}\right) U_{i, j}}}=\frac{\overline{\bar{U}}_{i, j+1}-\overline{\bar{U}}_{i, j}}{k} .
\end{array}
$$

For $\left(D_{x}\right) \tilde{U}_{i j}$ at $p$ we have:

$$
\begin{array}{ll}
\underline{\left(D_{x}\right) U_{i, j}}=\frac{\underline{U}_{i, j}-\underline{U}_{i-1, j}}{h} ; & \overline{\left(D_{x}\right) U_{i, j}}=\frac{\bar{U}_{i, j}-\bar{U}_{i-1, j}}{h} ; \\
\underline{\underline{\left(D_{x}\right) U_{i, j}}}=\frac{\underline{\underline{U}}_{i, j}-\underline{\underline{U}}_{i-1, j}}{h} ; & \overline{\overline{\left(D_{x}\right) U_{i, j}}}=\frac{\overline{\bar{U}}_{i, j}-\overline{\bar{U}}_{i-1, j}}{h} .
\end{array}
$$

Therefore, the parametric form of one dimensional transport equation $\left(D_{t}\right) \tilde{U}_{i, j}=b\left(D_{x}\right) \tilde{U}_{i, j}$ is: 


$$
\begin{aligned}
& \frac{\underline{U}_{i, j+1}-\underline{U}_{i, j}}{k}=b\left(\frac{\underline{U}_{i, j}-\underline{U}_{i-1, j}}{h}\right), \\
& \frac{\bar{U}_{i, j+1}-\bar{U}_{i, j}}{k}=b\left(\frac{\bar{U}_{i, j}-\bar{U}_{i-1, j}}{h}\right), \\
& \frac{\underline{\underline{U}}_{i, j+1}-\underline{\underline{U}}_{i, j}}{k}=b\left(\frac{\underline{\underline{U}}_{i, j}-\underline{\underline{U}}_{i-1, j}}{h}\right), \\
& \frac{\overline{\bar{U}}_{i, j+1}-\overline{\bar{U}}_{i, j}}{k}=b\left(\frac{\overline{\bar{U}}_{i, j}-\overline{\bar{U}}_{i-1, j}}{h}\right) .
\end{aligned}
$$

By (6.1), (6.2), (6.3) and (6.4) the following equation must hold:

$$
\begin{aligned}
& \underline{U}_{i, j+1}=\underline{U}_{i, j}+r\left(\underline{U}_{i, j}-\underline{U}_{i-1, j}\right), \\
& \bar{U}_{i, j+1}=\bar{U}_{i, j}+r\left(\bar{U}_{i, j}-\bar{U}_{i-1, j}\right), \\
& \underline{\underline{U}}_{i, j+1}=\underline{\underline{U}}_{i, j}+r\left(\underline{\underline{U}}_{i, j}-\underline{\underline{U}}_{i-1, j}\right), \\
& \overline{\bar{U}}_{i, j+1}=\underline{\underline{U}}_{i, j}+r\left(\underline{\underline{U}}_{i, j}-\overline{\bar{U}}_{i-1, j}\right),
\end{aligned}
$$

where $x_{i}=i h,(i=0,1,2,3, \ldots, n), y_{j}=j k,(j=0,1,2, \ldots, n)$ and $r=\frac{k b}{h}$.
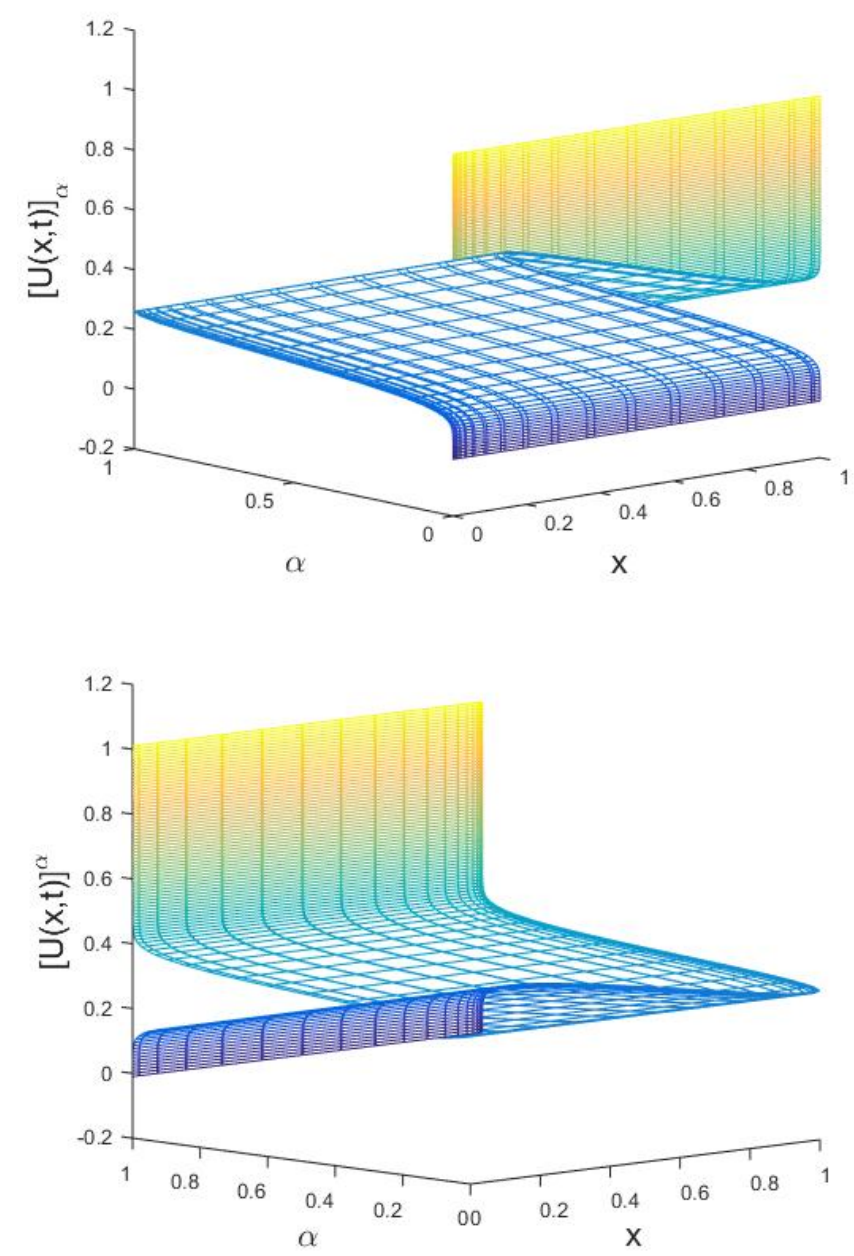

Figure 1: The 3D of intuitionistic fuzzy exact and approach solution at $h=0.01, k=0.009$. 
Figure 1 presents the $\alpha$-cuts of intuitionistic fuzzy solutions in 3D, i.e., the curves of the solutions correspond to the upper and lower bounded $[U(x, t)]^{\alpha}$ and $[U(x, t)]_{\alpha}$.

\section{Conclusion}

In this paper, we have studied the existence result for a solution of intuitionistic fuzzy transport equation in homogeneous and non-homogeneous cases. Also, we showed the solution for advection equation by the finite difference method that gave a good approximation for the solution.

\section{References}

[1] Atanassov, K. T. (1983). Intuitionistic Fuzzy Sets. VII ITKR Session, Sofia, 20-23.

[2] Atanassov, K. T. (1994). New operations defined over the intuitionistic fuzzy sets. Fuzzy Sets and Systems, 61(2), 137-142.

[3] Atanassov, K. T. (2017). Intuitionistic Fuzzy Logics. Studies in Fuzziness and Soft Computing, 351, Springer, Cham.

[4] Bede, B., Rudas, I. J., \& Bencsik, A. (2007). First order linear differential equations under generalized differentiability. Information Sciences, 177, 1648-1662.

[5] Bertone, A. M., Jafelice, R. M., Barros, L. C., \& Bassanezi, R. C. (2013). On fuzzy solutions for partial differential equations. Fuzzy Sets and Systems, 210, 68-80.

[6] Melliani, S., Ettoussi, R., Elomari, M., \& Chadli, L. S. (2015). Solution of intuitionistic fuzzy differential equations by successive approximations method. Notes on Intuitionistic Fuzzy Sets, 21(2), 51-62.

[7] Melliani, S., Elomari, M., Chadli, L. S., \& Ettoussi, R. (2015). Intuitionistic fuzzy metric space. Notes on Intuitionistic Fuzzy Sets, 21(1), 43-53.

[8] Melliani, S., Belhallaj, Z., Elomari, M., \& Chadli, L. S. (2021). Approximate Solution of Intuitionistic Fuzzy Differential Equations with the Linear Differential Operator by the Homotopy Analysis Method. Advances in Fuzzy Systems.

[9] Prakash, J., Arun Balaji, R., \& Wakgari, D. (2019). A Method for solving fuzzy partial differential equation by fuzzy separation. International Research Journal of Engineering and Technology, 6(1), 77-86.

[10] Seikkala, S. (1987). On the fuzzy initial value problem. Fuzzy Sets and Systems, 24, 319-330.

[11] Zadeh, L. A. (1965). Fuzzy sets. Information and Control, 8(3), 338-353. 